\title{
The application of tetracycline- regulated gene expression systems in the validation of novel drug targets in Mycobacterium tuberculosis
}

OPEN ACCESS

Edited by:

Teresa M. Coque, Hospital Universitario Ramón y Cajal,

Spain

Reviewed by:

Darío García De Viedma,

Hospital Gregorio Marañón, Spain

Jesus Blazquez,

Fundación Pública Andaluza para la Gestión de la Investigación en Salud

de Sevilla, Spain

*Correspondence:

Joanna C. Evans and

Valerie Mizrahi,

South African Medical Research

Council/National Health Laboratory

Service/University of Cape Town

Molecular Mycobacteriology

Research Unit, Anzio Road,

Observatory 7925, Cape Town, South

Africa

joanna.evans@uct.ac.za; valerie.mizrahi@uct.ac.za

Specialty section:

This article was submitted to

Antimicrobials, Resistance and Chemotherapy,

a section of the journal

Frontiers in Microbiology

Received: 02 June 2015

Accepted: 23 July 2015

Published: 04 August 2015

Citation:

Evans JC and Mizrahi V (2015)

The application of tetracyclineregulated gene expression systems in the validation of novel drug targets

in Mycobacterium tuberculosis.

Front. Microbiol. 6:812.

doi: 10.3389/fmicb.2015.00812

\section{Joanna C. Evans ${ }^{1,2 *}$ and Valerie Mizrahi, ${ }^{1,2 *}$}

${ }^{1}$ South African Medical Research Council/National Health Laboratory Service/University of Cape Town Molecular Mycobacteriology Research Unit, Cape Town, South Africa, ${ }^{2}$ DST/NRF Centre of Excellence for Biomedical TB Research, Institute of Infectious Disease and Molecular Medicine and Division of Medical Microbiology, Faculty of Health Sciences, University of Cape Town, Cape Town, South Africa

Although efforts to identify novel therapies for the treatment of tuberculosis have led to the identification of several promising drug candidates, the identification of highquality hits from conventional whole-cell screens remains disappointingly low. The elucidation of the genome sequence of Mycobacterium tuberculosis (Mtb) facilitated a shift to target-based approaches to drug design but these efforts have proven largely unsuccessful. More recently, regulated gene expression systems that enable dosedependent modulation of gene expression have been applied in target validation to evaluate the requirement of individual genes for the growth of $M$ tb both in vitro and in vivo. Notably, these systems can also provide a measure of the extent to which putative targets must be depleted in order to manifest a growth inhibitory phenotype. Additionally, the successful implementation of Mtb strains engineered to under-express specific molecular targets in whole-cell screens has enabled the simultaneous identification of cell-permeant inhibitors with defined mechanisms of action. Here, we review the application of tetracycline-regulated gene expression systems in the validation of novel drug targets in Mtb, highlighting both the strengths and limitations associated with this approach to target validation.

Keywords: Mycobacterium tuberculosis, drug discovery, regulated gene expression, hypomorphs, target validation, target-based whole cell screening

Tuberculosis (TB) remains a major global health crisis, with 9 million new cases and 1.5 million deaths attributable to this disease in 2013 (WHO, 2014). The treatment of TB is challenging, requiring combination therapy with rifampicin, isoniazid, pyrazinamide, and ethambutol for 2 months, followed by an additional 4 months of treatment with rifampicin and isoniazid. Despite this extended period of treatment, however, strains of Mycobacterium tuberculosis (Mtb) resistant to rifampicin and isoniazid, defined as multidrug-resistant (MDR), continue to emerge, with an estimated 480,000 cases of MDR-TB identified in 2013 (WHO, 2014). The more recent emergence of MDR strains with additional resistance to fluoroquinolones and at least one of the injectable aminoglycosides (amikacin, kanamycin, or capreomycin) - termed extensively drugresistant (XDR) - has resulted in the need to implement therapies that must be continued for up to 24 months, and involve the use of more costly, less effective, and more toxic second-line 
drugs. Thus, the emergence and spread of drug-resistant strains of $M t b$ has complicated the already daunting challenge of global TB control, and has underscored the urgent need to identify new anti-tubercular agents with novel mechanisms of action (Zumla et al., 2013). This need has driven the establishment of a TB drug pipeline, which is populated with a number of promising candidates and novel drug combinations at various stages of clinical development. However, the typically high attrition rate for drugs in clinical development, coupled with specific weaknesses in the TB drug pipeline - in particular, the paucity of candidates in the early stages of clinical validation makes the development of new drug regimens for the treatment of drug-resistant as well as drug-susceptible TB critically reliant upon replenishment of the pipeline through the identification of high-quality "hit" compounds and novel targets by innovative TB drug discovery programs.

In the target-based approach to $\mathrm{TB}$ drug discovery, compounds that inhibit the biochemical function of the target of interest are identified by high-throughput screening (HTS) of compound libraries or by structure-guided drug design (Hung et al., 2009; Willand et al., 2009; Krieger et al., 2012). While this approach has yielded compounds with potent inhibitory activity against validated targets, the compounds frequently fail to display whole-cell activity against live bacilli (Payne et al., 2007; Cole and Riccardi, 2011; Xu et al., 2014). This problem is commonly attributed to issues of permeation, metabolism, and efflux, all of which are likely exacerbated by the comparatively complex intracellular environment in which the drug-target interaction must occur in a cell-based assay. In the alternative, phenotypic approach, cell-based HTS of compound libraries has been used to identify inhibitors with whole-cell activity against $M t b$ in vitro. This approach has been considerably more successful (Stover et al., 2000; Matsumoto et al., 2006; Makarov et al., 2009; Pethe et al., 2013; Manjunatha et al., 2015), and led to the recent development of bedaquiline (Sirturo; Andries et al., 2005), the first FDA-approved drug for the treatment of TB in over 40 years (Cohen, 2013); delamanid (Deltyba), which received conditional approval by the European Medicines Agency in 2013 and is being evaluated in a phase 3 trial for the treatment of MDR-TB (Gler et al., 2012); and PA-824 (pretomanid), which has shown promise as part of a new threedrug regimen (Dawson et al., 2015). Importantly, hit compounds discovered through phenotypic screens have been used to identify novel targets in previously unexploited pathways, such as AtpE (target of bedaquiline) and QcrB (targeted by imidazopyridines other chemotypes) in energy metabolism, and DprE1 (benzothiazinones and other chemotypes) in arabinan biosynthesis (Andries et al., 2005; Petrella et al., 2006; Makarov et al., 2009; Magnet et al., 2010; Abrahams et al., 2012b; Stanley et al., 2012; Pethe et al., 2013; Preiss et al., 2015). However, deducing mechanisms of action of inhibitors with whole-cell activity can often be challenging, particularly if the inhibitory effects are pleiotropic (Kohanski et al., 2008) or if the target is not a protein (Ling et al., 2015).

A fundamental requirement of a desirable antimicrobial drug target is that it should have an essential, and preferably nonredundant, function in growth and pathogenesis of the organism.
The study of (conditionally) essential gene function in bacteria has been revolutionized by the development of regulated gene expression systems, which enable the expression of a target gene to be controlled in a manner that depends upon the level of an inducer. A number of such systems have been developed for use in mycobacteria, and have been employed to investigate the roles of a wide variety of genes essential and conditionally essential for bacillary growth, physiology, and metabolism (Gomez and Bishai, 2000; Greendyke et al., 2002; Dziadek, 2003; Trauner et al., 2012; Ventura et al., 2013; Chuang et al., 2015). In this review, we focus on the use of tetracycline (Tet)-regulated gene expression systems in $M t b$, specifically in the context of target validation. We highlight the technical advancements that have been made, and describe the application of conditional mutants of $M t b$ in the validation of novel drug targets, and in target-based whole-cell screening (TB-WCS), in which phenotypic and target-based approaches are combined to identify target- and pathway-selective compounds with wholecell activity.

\section{Tet-Regulated Genetic Switches for Controlling Gene Expression in Mtb}

Regulated gene expression systems have been extensively characterized and utilized in both Gram-positive and Gramnegative bacteria (Bertram and Hillen, 2008), yet limited knowledge of the transcriptional regulatory mechanisms and machinery complicated the development of such systems for use in mycobacteria until relatively recently. The first successful demonstration of dose-responsive gene expression in mycobacteria was achieved by expressing the $35 \mathrm{kDa}$ antigen of $M$. leprae from the acetamide-inducible promoter of Mycobacterium smegmatis (Msm; Parish et al., 1997; Triccas et al., 1998). This system has since been used successfully to express several mycobacterial proteins in Msm (Gomez and Bishai, 2000; Ojha et al., 2000; Parish et al., 2001; Daugelat et al., 2003; Dziadek, 2003; Ahidjo et al., 2011; Lee et al., 2014); however, instability in $M t b$ has limited its utility in this species (Brown and Parish, 2006). Several other regulated gene expression systems, including those mediated by Tet, nitrile, and pristinamycin, have subsequently been developed for use in mycobacteria (Table 1A); these have been reviewed elsewhere (Schnappinger and Ehrt, 2014) so will not be described here. In this review, we focus specifically on the Tet-regulated gene expression systems (Table 1B; Blokpoel et al., 2005; Carroll et al., 2005; Ehrt et al., 2005), which have been widely applied, and whose utility in TB drug discovery is enhanced by the fact that they can be used to regulate gene expression in animal models of infection.

The mechanism of transcriptional regulation using Tetcontrolled genetic switches is based on that of a family of Tet-exporting proteins that mediate resistance to Tet in Gramnegative organisms (Berens and Hillen, 2003). In the absence of Tet, Tet repressor (TetR) proteins bind tightly to the Tet operators $(t e t O)$ located within the promoter of tetA, encoding the Tet-exporting protein, thereby suppressing tetA expression 
TABLE 1 | Regulated gene expression systems developed for use in Mycobacterium tuberculosis (Mtb).

\begin{tabular}{|c|c|c|c|}
\hline \multirow[t]{2}{*}{ Expression system } & \multicolumn{2}{|c|}{ Regulatory components } & \multirow[t]{2}{*}{ Reference } \\
\hline & Promoter & Regulator & \\
\hline \multicolumn{4}{|c|}{ (A) Gene regulation systems utilized in mycobacteria } \\
\hline Tet-ON & $\begin{array}{l}\text { Tet-inducible promoter of tetA from } \\
\text { Corynebacterium glutamicum }\end{array}$ & TetR repressor from C. glutamicum & Blokpoel et al. (2005) \\
\hline Tet-ON & $\begin{array}{l}\text { Tet-inducible promoter } P_{x y l} \text { from } \\
\text { Bacillus subtilis }\end{array}$ & $\begin{array}{l}\text { Tn10-derived TetR repressor from } \\
\text { Escherichia coli }\end{array}$ & Carroll et al. (2005) \\
\hline NitR & $\begin{array}{l}\text { Nitrile-inducible promoter of nitA from } \\
\text { Rhodococcus rhodochrous }\end{array}$ & $\begin{array}{l}\text { NitR regulator, both from } \\
R . \text { rhodochrous }\end{array}$ & Pandey et al. (2009) \\
\hline Pip-ON & $\begin{array}{l}\text { Pristinamycin-inducible promoter, } \mathrm{P}_{\mathrm{ptr}} \\
\text { from Streptomyces pristinaespiralis }\end{array}$ & $\begin{array}{l}\text { Pristinamycin-responsive repressor, } \\
\text { Pip, from S. coelicolor }\end{array}$ & Forti et al. (2009) \\
\hline Tet/Pip-OFF & $\begin{array}{l}\text { Pristinamycin-inducible promoter, } \mathrm{P}_{\mathrm{ptr}} \text {, } \\
\text { from S. pristinaespiralis }\end{array}$ & $\begin{array}{l}\text { Pristinamycin-responsive repressor, } \\
\text { Pip, from S. coelicolor under control of } \\
\text { Tet-inducible promoter, } P_{\text {furA102 }} \text { tetO }\end{array}$ & Boldrin et al. (2010) \\
\hline \multicolumn{4}{|c|}{ (B) Tetracycline (Tet)-regulated gene expression systems optimized for use in mycobacterial gene regulation } \\
\hline Tet-ON & $\begin{array}{l}\text { Tet-inducible mycobacterial promoter, } \\
\mathrm{P}_{\mathrm{myc} 1} \text { tetO }\end{array}$ & $\begin{array}{l}\text { Tn10-derived TetR repressor from } \\
\text { E. coli }\end{array}$ & Ehrt et al. (2005) \\
\hline Tet-OFF & $\begin{array}{l}\text { Tet-inducible mycobacterial promoter, } \\
\mathrm{P}_{\mathrm{myc} 1} \text { tetO }\end{array}$ & $\begin{array}{l}\text { Tn10-derived reverse TetR inducer from } \\
\text { E. coli }\end{array}$ & Guo et al. (2007) \\
\hline
\end{tabular}

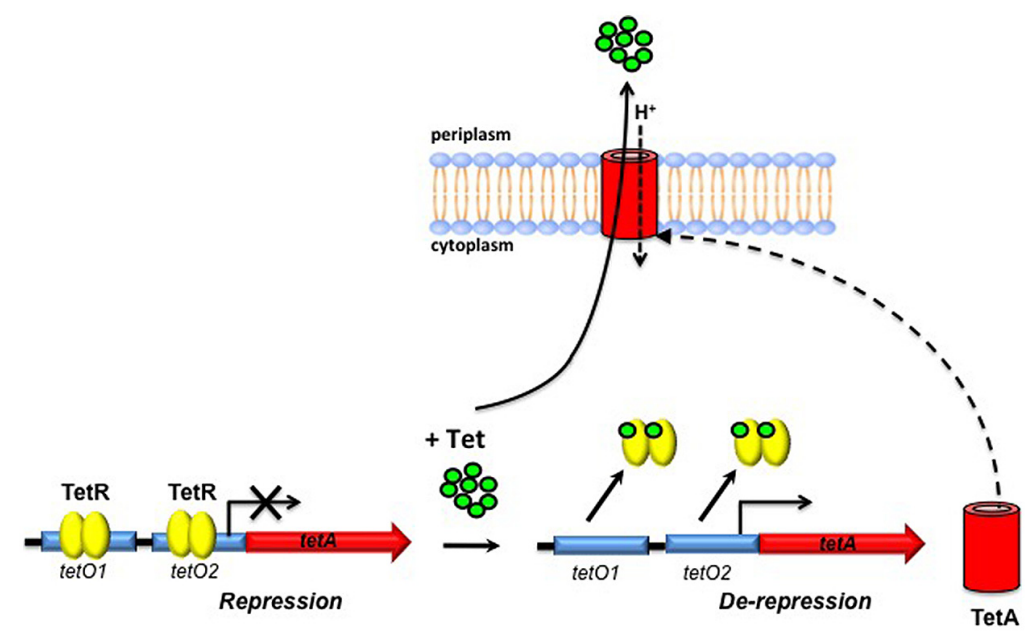

FIGURE 1 | Schematic representation of tetracycline (Tet) export in Gram-negative bacteria. In the absence of Tet, Tet repressor (TetR) dimers bind to Tet operators (tetO) located upstream of tet $A$, encoding the Tet-exporting protein, repressing its transcription. Upon introduction of anhydrotetracycline (ATC) and its subsequent binding to TetR, a conformational change occurs that results in dissociation of TetR from tetO, enabling Tet-mediated transcription of tetA to occur. Tet is then transported across the cytoplasmic membrane by TetA.
(Figure 1; Berens and Hillen, 2003). Owing to the substantially higher affinity of TetR for Tet than for the ribosome (Lederer et al., 1996), the introduction of Tet causes a conformational change that results in dissociation of TetR from tet $O$, leading to de-repression of tet $A$ and ultimately to the efflux of Tet (Berens and Hillen, 2003). By replacement of the native Mtb promoter of a gene of interest with a Tet-regulatable promoter, and introduction of TetR on a replicating or integrative plasmid, dose-dependent induction of gene expression can be mediated through the addition of varying concentrations of the less toxic derivative, anhydrotetracycline (ATc; Blokpoel et al., 2005; Carroll et al., 2005; Ehrt et al., 2005). Since it is important that introduction of the inducer has a minimal effect on the expression of other genes, an additional advantage of the Tetregulated system is that gene expression can be induced with ATc concentrations at least 10 -fold below the concentration required to inhibit the growth of Msm or Mtb (Ehrt and Schnappinger, 2006).

Conditional knockdown mutants - or "hypomorphs" - in which expression of the target gene is switched on upon addition of ATc are commonly referred to as "Tet-ON" mutants; in this configuration, removal of the inducer is required in order to silence target gene expression (Figure 2A). The dynamic range of the Tet-ON system can be expanded further by expression of TetR from either a strong promoter or an intermediatestrength promoter to generate hypomorphs in the Tet-ON 
A

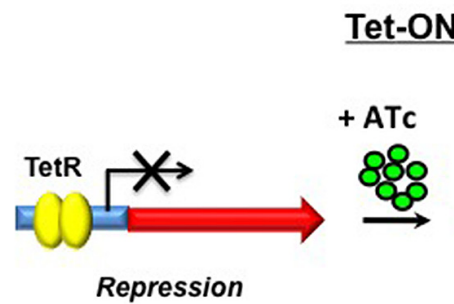

B
Tet-ON

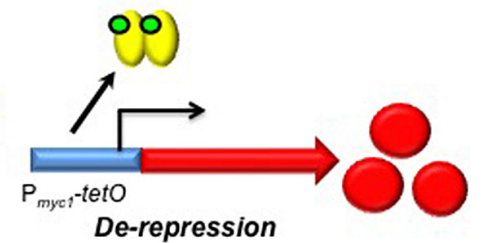

TARGET

Tet-OFF

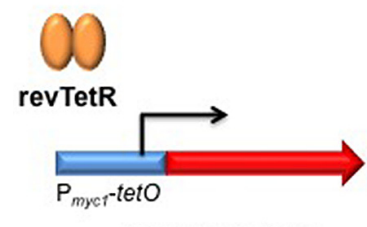

De-repression
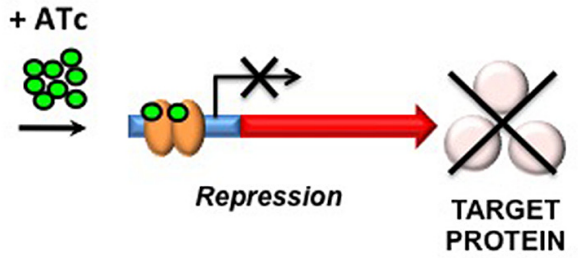

FIGURE 2 | Tetracycline-mediated gene regulation in Mycobacterium tuberculosis (Mtb). (A) In the Tet-ON configuration, TetR dimers bind to Tet operators (tetO) in the absence of ATc, repressing transcription. Upon introduction of ATc and its subsequent binding to TetR, a conformational change occurs that results in dissociation of TetR from tetO, enabling Tet-mediated transcription from $\mathrm{P}_{\mathrm{myc} 1}$ tetO to occur. (B) In the Tet-OFF configuration, introduction of ATc results in binding of revTetR to $\mathrm{P}_{\text {myc } 1}$ tetO, causing repression of transcription. TetR, Tet repressor; ATc, anhydrotetracycline; tetO, Tet operator; $\mathrm{P}_{\text {myc1 } 1}$ tetO, mycobacterial promoter with tetOs inserted; revTetR, reverse Tet repressor (Adapted from Guo et al., 2007). and Tet- $\mathrm{ON}_{M}$ configurations, respectively. This increases the flexibility of the system, enabling dose-dependent regulation of genes with widely differing levels of expression. Since the removal of ATc can be difficult to achieve in some experimental settings, the manipulation of Tet-dependent hypomorphs was greatly simplified by the development of a modified "Tet-OFF" system, which utilizes a mutated, "reverse" TetR (revTetR) that binds to tetO only in the presence of ATc (Guo et al., 2007). This system enables the generation of mutants in which target gene expression is repressed upon addition of the ATc inducer (Figure 2B). The regulatory capacity of both Tet-ON and TetOFF systems in mycobacteria has been enhanced by codon optimization of the genes encoding TetR and revTetR to allow increased expression in $M t b$ (Klotzsche et al., 2009). Together, these modifications have provided a means of evaluating the effects of transcriptional silencing of target genes on growth and viability of the organism.

\section{Application of Tet-Regulated Systems in Target Validation}

The utility of genetic switches for regulating gene expression in $M t b$ was first demonstrated by the generation of a tryptophan auxotroph following Tet-mediated transcriptional silencing of $\operatorname{trpD}$ (Carroll et al., 2005). Transcriptional silencing has subsequently been used to confirm the in vitro essentiality of a variety of genes in $M t b$, thus providing preliminary genetic validation of the encoded proteins as potential drug targets. These include, among others, fatty-acid-coenzyme A (CoA) ligase (fadD32; Carroll et al., 2011), diaminopimelate decarboxylase
(lysA; Abrahams et al., 2012a), Clp protease (clpP1; Ollinger et al., 2012b), pantothenate synthetase (panC; Abrahams et al., 2012a), thymidylate synthase (thyX; Singh et al., 2015), ribonucleotide reductase ( $n r d F 2$; Singh et al., 2015), topoisomerase I (Rv3646c; Ahmed et al., 2014) and seven genes (dprE1, dprE2, ubiA, $\operatorname{prs} A, R v 2361 c$, tkt, and rpiB) involved in decaprenyl-phosphoD-arabinofuranose (DPA) biosynthesis in Mtb (Kolly et al., 2014a).

A major advantage of Tet-regulated systems is that the ability of ATc to diffuse across biological membranes also allows these systems to be used to regulate expression of $M t b$ genes in infected macrophages (Blokpoel et al., 2005; Ehrt et al., 2005). Furthermore, the pharmacokinetic properties of ATc and its analog, doxycycline (doxy), permit administration of this inducer to animals at doses required to successfully regulate gene expression in vivo (Ehrt and Schnappinger, 2006) without compromising the growth of wildtype bacilli (Gandotra et al., 2007; Blumenthal et al., 2010). Since the conditions under which $M t b$ can be cultured in vitro are unlikely to fully recapitulate the environment encountered in the host during infection, this can have significant effects on the physiology, metabolism and global transcriptional response of the bacilli and, hence, on the relevance of the conclusions drawn from such analyses (Bloch and Segal, 1956; Schnappinger et al., 2003; Pethe et al., 2010; Griffin et al., 2012). The development of the Tet-system therefore represented a major breakthrough in mycobacterial genetics by providing a means to identify genes that are (conditionally) essential for growth of $M t b$ in vivo (Gandotra et al., 2007). Silencing of $\operatorname{prcBA}$, encoding the $\alpha$ and $\beta$ subunits of the $M t b$ 20S proteasome, either immediately post-infection, or during the chronic stage of infection (i.e., following onset of the acquired immune response), 
resulted in a decline in bacillary load in the lungs and spleens of mice, indicating a role for the proteasome in both growth and persistence of Mtb in vivo (Gandotra et al., 2007). The successful demonstration of the utility of this approach paved the way for a slew of subsequent studies aimed at investigating the requirement of several $M t b$ genes for growth during different phases of infection (Table 2 ), simultaneously providing genetic validation of many of these as potential drug targets. Although perhaps not of direct relevance to the physiology of $M t b$ itself, it is worth noting that the selective inhibition of mitochondrial protein translation by Tets can lead to altered transcriptional profiles in eukaryotic cell lines and has minor effects on physiological fitness in mice (Moullan et al., 2015). This may, in turn, affect the contribution of the murine immune response to $M t b$ clearance.

An important caveat to the use of hypomorphs for confirming target essentiality both in vitro and in vivo is the potential for suppressors to emerge that are no longer responsive to the inducer (Stallings et al., 2009; Boldrin et al., 2010; Kolly et al., 2014a). The loss of inducer dependence of gene expression can arise through the acquisition of mutations in the regulatory network and/or loss of the transcriptional repressor as a consequence of the strong selective pressure conferred on $M t b$ by depletion of an essential cellular function. Since the emergence of suppressor mutants masks the effects of inducer-dependent transcriptional silencing, it is essential to include appropriate controls for detection of populations of bacilli that are no longer inducer-responsive when phenotypically characterizing hypomorphs produced using these systems.

\section{Assessing Target Vulnerability by Tet-Mediated Transcriptional Silencing}

Genome-wide transposon mutagenesis has played a critical role in defining the genetic requirements for growth and viability of $M t b$ under various conditions (Sassetti and Rubin, 2003;
Sassetti et al., 2003; Rengarajan et al., 2005; Beste et al., 2009; Griffin et al., 2011). However, the complete elimination of biochemical function resulting from insertional inactivation of a gene encoding an essential cellular function is unlikely to be achieved by chemical inhibition of that function, particularly in the validation phase of drug discovery when inhibitory activity has not yet been optimized (Barry et al., 2009). Recent target validation efforts have thus focused on determining target "vulnerability," which can be operationally defined as the extent to which the activity/function of a target must be reduced in order to manifest in a growth phenotype (Figure 3A). Given increasing evidence suggesting that targets requiring incomplete inhibition in order to confer a lethal phenotype may be more valuable (Barry et al., 2009; Kim et al., 2011; Wei et al., 2011), the ability to silence gene expression in a dose-dependent manner provides a potentially powerful means to infer the vulnerability of a given target.

The construction of hypomorphs by transcriptional silencing has allowed target vulnerability to be analyzed by quantifying the relative level of transcript depletion associated with bacterial growth inhibition and/or loss of viability (Gandotra et al., 2007; Forti et al., 2009; Boldrin et al., 2010; Carroll et al., 2011; Kolly et al., 2014a). However, the kinetics of depletion of a protein by transcriptional silencing depend upon the specific protein half-life. Therefore, it is important to monitor the extent of protein depletion and its association with bacterial growth and/or viability during the course of a silencing experiment as silencing of genes encoding long-lived proteins may result in an underestimation of the vulnerability of those targets. It is also important to consider the relative strengths of the inducible promoter vs. the native promoter of the gene of interest when constructing hypomorphs. In cases where the target protein is in low abundance in wildtype bacilli, promoter replacement may result in the target being expressed at a higher level from the regulated promoter than from its native promoter under conditions of maximal de-repression. In such cases, efficient

TABLE 2 | Targets validated by Tet-mediated control of $M$ tb gene expression in vivo.

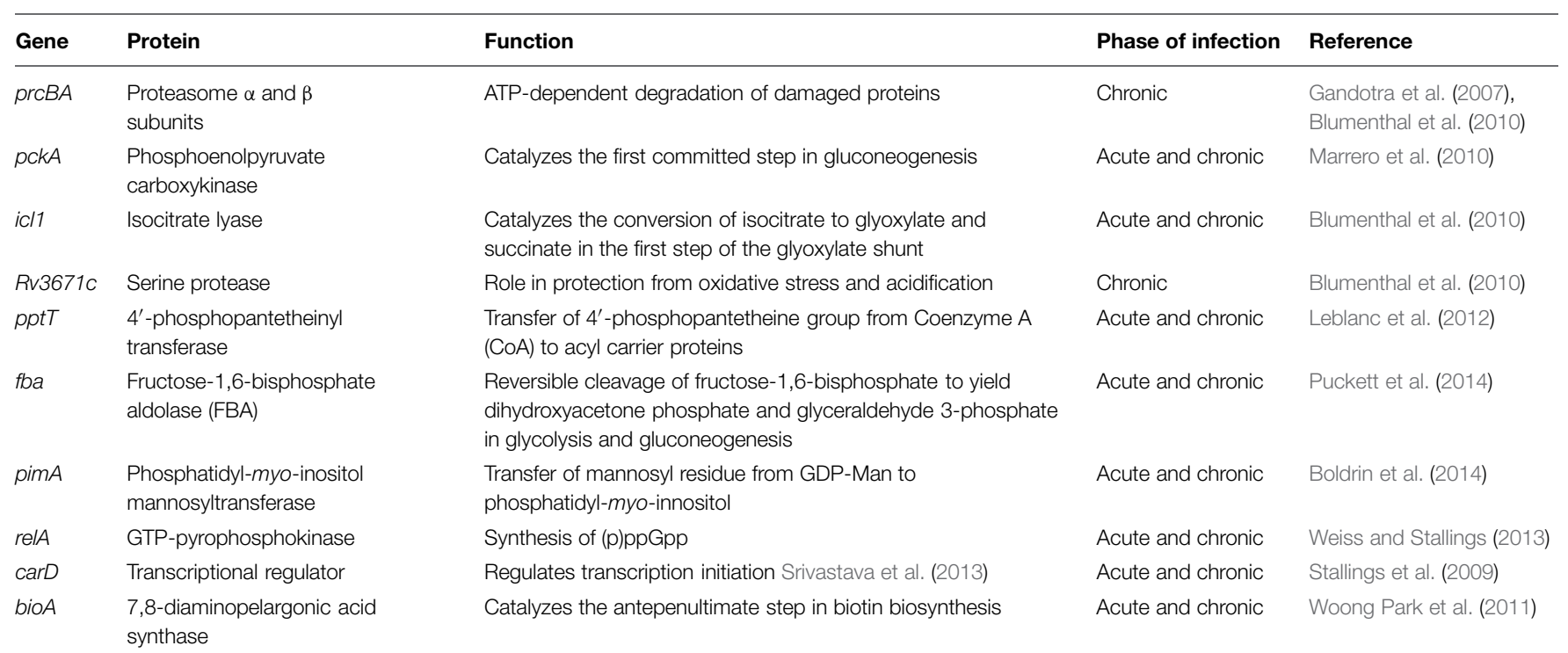


A

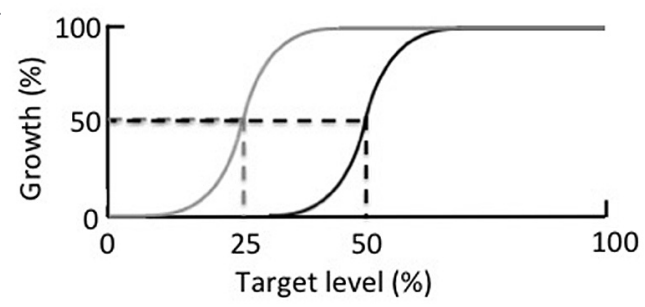

B

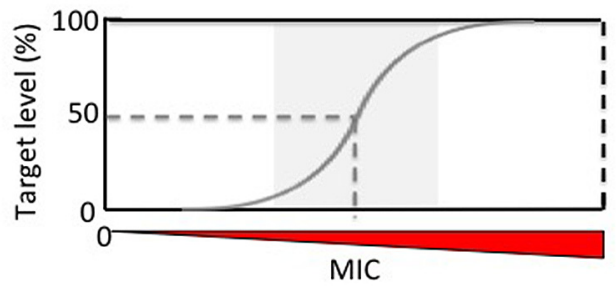

FIGURE 3 | The application of hypomorphs in (A) assessing target vulnerability and (B) expanding antitubercular chemical space.

(A) Dose-dependent silencing of a target gene enables determination of the extent to which that target must be depleted in order to result in growth inhibition. A target that requires only $50 \%$ depletion in order to reduce $50 \%$ of bacterial growth (black line) is considered more vulnerable that one that requires $75 \%$ depletion to achieve the same effect (gray line). (B) Reduction of the absolute concentration of a target by conditional knockdown (gray line) relative to wildtype levels (black line) facilitates the identification of on-target inhibitors by lowering the minimum inhibitory concentration (MIC).

silencing is unlikely to be achieved (Ehrt and Schnappinger, 2006; Carroll et al., 2011). The converse may also occur, as observed in the case of icl1, which encodes isocitrate lyase, a key enzyme in the glyoxylate shunt. Icl1 is conditionally essential for growth of $M t b$ on fatty acids and is transcriptionally upregulated when the organism is cultured on acetate (Munoz-Elias and McKinney, 2005; Blumenthal et al., 2010). Replacement of the native icl1 promoter with the Tet-regulated promoter, $\mathrm{P}_{\text {mycl }}$ tet $O$, resulted in a 16 -fold increase in sensitivity of $M t b$ to the Icl inhibitor, 3-nitropropionate (3-NP), when grown on acetate, most likely reflecting a significantly lower level of icll expression by the Tet-regulated promoter than the native (fatty-acid-inducible) promoter (Abrahams et al., 2012a).

Transcriptional silencing has been used to infer relative invulnerability for a number of potential drug targets for which small molecule inhibitors have been developed. For example, pantothenate kinase (PanK), the enzyme that catalyzes the first, and rate-limiting, step in the conversion of pantothenate (vitamin B5) into CoA (Das et al., 2006), has attracted considerable attention as a TB drug target (Begley et al., 2001; Spry et al., 2008; Moolman et al., 2014). Although essential for growth of Mtb in vitro (Sassetti et al., 2003; Griffin et al., 2011; Reddy et al., 2014), and despite the fact that transcriptional silencing of panK (coaA) resulted in depletion of PanK protein to below the limit of detection, growth of $M t b$ in liquid culture and in vivo was not impeded (Reddy et al., 2014). A similar approach was used to show that $>95 \%$ depletion of another enzyme in the CoA biosynthesis pathway, pantothenate synthetase ( $\operatorname{PanC}$ ), is required to effect complete growth inhibition of $\mathrm{Mtb}$ in vitro
(Abrahams et al., 2012a). Likewise, in the case of the biotin biosynthesis enzyme, BioA, which is essential for growth in vitro and persistence of $M t b$ in mice (Sassetti et al., 2003; Woong Park et al., 2011), >95\% depletion of the protein was required in order to completely inhibit $M t b$ growth (Woong Park et al., 2011). These results suggest that PanK, PanC, and BioA represent relatively invulnerable targets in $M t b$ and, since complete inactivation of a target's function by a small molecule inhibitor is not readily achievable, this may account, at least in part, for the lack of whole-cell activity of known inhibitors of these enzymes against wildtype $M t b$ (Ciulli et al., 2008; Abrahams et al., 2012a; Bjorkelid et al., 2013; Reddy et al., 2014; Park et al., 2015). However, it is important to recognize that while knowledge of the relative vulnerabilities of potential targets as inferred from target depletion studies - can be useful in terms of guiding target-based approaches to drug design, this notion is of less relevance in the case of an inhibitor that binds irreversibly to, or induces a conformational change in, its target. This point is illustrated in the case of BioA and the antibiotic, amiclenomycin: despite the relative invulnerability of BioA suggested by transcriptional silencing, amiclenomycin is able to bind covalently to this protein, thereby rendering it irreversibly inactive, which results in growth inhibition of $M t b$ (Sandmark et al., 2002; Woong Park et al., 2011).

\section{A Pathway Approach to Prioritizing Novel Drug Targets}

There is renewed interest in targeting core metabolic pathways in Mtb for drug discovery (Murima et al., 2014; Puckett et al., 2014; Trujillo et al., 2014). In this context, the ability to rank enzymes in a prioritized metabolic or biosynthetic pathway on the basis of relative vulnerability can be particularly useful for focusing efforts. An excellent example of the value of this approach comes from a study on the biosynthesis of DPA, a precursor of the arabinogalactan component the mycobacterial cell wall (Kolly et al., 2014a). DPA biosynthesis in $M t b$ takes place in five steps involving eight genes (Wolucka, 2008; Kolly et al., 2014a), all but one of which are predicted to be required for bacillary growth (Sassetti et al., 2003; Griffin et al., 2011). The epimerase DprE1, which catalyzes the fifth and final step in the DPA biosynthesis pathway in conjunction with DprE2 (Wolucka, 2008; Kolly et al., 2014a), has been identified as the target of several new classes of inhibitors that display potent anti-tubercular activity, and has been rigorously validated as a novel TB drug target (Christophe et al., 2009; Makarov et al., 2009; Magnet et al., 2010; Stanley et al., 2012; Wang et al., 2013). To identify the steps in the DPA pathway that are most vulnerable to target depletion, Kolly et al. (2014a) generated conditional knockdown mutants in every gene and compared the effects of transcriptional silencing of each on the growth and viability of $M t b$ in vitro and in macrophages.

This study revealed that, in addition to $d p r E 1$, transcriptional silencing of $u b i A$, encoding the decaprenyl-phosphate phosphoribosyltransferase UbiA, and prsA, encoding the phosphoribosyl pyrophosphate (PRPP) synthetase PrsA, exhibited the most profound growth inhibitory effects, with 
depletion of each resulting in a loss of $M t b$ viability in vitro and in macrophages. While the cidality associated with transcriptional silencing of $d r p E 1$ and $u b i A$ in vitro was shown to occur as a result of induction of cell lysis, silencing of prs $A$ resulted in reduced viability through cell implosion, without lysis occurring. Interestingly, although cell death occurred more slowly upon depletion of UbiA or PrsA than DprE1, the decrease in nucleic acid and protein synthesis observed as a consequence of PrsA depletion suggested that the bactericidal effects of PrsA inhibition are likely to be pleiotropic, thus identifying this enzyme as another promising a target in the DPA pathway. However, in an exciting new development, DprE1 was shown to occupy an extracytoplasmic location in $M t b$, suggesting that cellular localization may be a major determinant of the vulnerability of DprE1 to chemical inhibition, accounting for the potency of the many inhibitors that act on this target (Brecik et al., 2015).

\section{Target Depletion by Tet-Mediated Regulated Protein Degradation}

To overcome the limitations associated with using promoter replacement mutants for target depletion by transcriptional silencing, powerful new methods have been devised to enable regulated protein degradation in mycobacteria as an alternative approach (Kim et al., 2011; Wei et al., 2011). Although only reported thus far in Msm, this method nonetheless warrants discussion here owing to its obvious applicability in the validation of novel targets in $M t b$. Proteolytic degradation complexes play an important role in protein quality control in bacteria by recognizing and degrading misfolded proteins. In $M t b$, the ATPdependent intracellular ClpXP proteolytic complex, which is essential for growth in vitro (Sassetti et al., 2003; Raju et al., 2012) and during infection (Raju et al., 2012), mediates degradation of SsrA-tagged proteins (Figure 4A; Raju et al., 2012). The recognition of aberrant proteins by ClpXP is further enhanced by binding of an adapter protein, SspB, to SsrA-tagged proteins (Levchenko et al., 2000). Mutation of the ClpX-binding region of the SsrA tag to generate a modified tag, DAS+4, which carries a terminal DAS sequence in place of the LAA sequence in wildtype SsrA, weakens the interaction between ClpX and the targeted protein, resulting in SspB-dependent proteolysis of the tagged protein (Figure 4B; Griffith and Grossman, 2008). Through elegant manipulation of the mycobacterial Clp protease system, Kim et al. (2011) demonstrated that degradation of DAS+4-tagged proteins could be induced by TetR-mediated induction of SspB in Msm (Figure 4C). Using this system, dose-dependent Tet-regulated depletion of RpoB was achieved by insertion of a DAS +4 tag at the $3^{\prime}$ end of the $r p o B$ gene, which encodes the target of the first-line antiTB drug, rifampicin. Importantly, targeted degradation of RpoB had the same bactericidal effect on Msm as treatment with rifampicin.

A variation of this approach was subsequently employed to compare the effects of regulated degradation of $\mathrm{RpoB}$ and five other well-validated antimycobacterial drug targets -
GyrA, the target of the fluoroquinolones; Alr, a target of D-cycloserine; dihydrofolate reductase (DHFR), the target of trimethoprim; InhA, the target of isoniazid; and KasA, the target of thiolactomycin - on the growth and viability of Msm (Wei et al., 2011). This study revealed that the rates of depletion of the six targets varied considerably (Wei et al., 2011): while InhA, Alr, and DHFR were undetectable following $3 \mathrm{~h}$ of incubation in the presence of the inducer, little effect was seen on levels of RpoB, GyrA, and KasA up to $6 \mathrm{~h}$ post-treatment. Moreover, the degree of protein depletion observed did not correlate with bacterial survival: whereas $80 \%$ reduction of RpoB was sufficient to arrest the growth of $M s m,>97 \%$ depletion of DHFR and Alr - as measured by immunoblotting and confirmed by enzyme activity analysis - had only modest effects on growth (Wei et al., 2011). Subsequent to this landmark study, regulated protein degradation has been use to validate a number of other drug targets in $M s m$, including those involved in nicotinamide adenine dinucleotide (NAD) biosynthesis (Rodionova et al., 2014), peptidoglycan biosynthesis (Gee et al., 2012) and intracellular protein degradation (Raju et al., 2012). Importantly, however, the varying effects of depleting pharmacologically validated targets of known TB drugs on $M t b$ viability calls for caution when using the outcome of regulated protein degradation studies as a primary or sole criterion for ranking, prioritizing or discarding novel drug targets.

\section{Tightened Regulation using a Tet-Mediated Dual Control System and its Application in the Validation of Persistence Targets}

Most efforts in TB drug discovery remain centered on identifying inhibitors of cellular processes required for bacillary growth under various conditions (Makarov et al., 2009; Hartkoorn et al., 2012; Blanco et al., 2015; Manjunatha et al., 2015). However, the critical role that populations of bacilli that are non-replicating or slowly replicating are thought to play in TB disease (Barry et al., 2009; Rittershaus et al., 2013) has underscored the need to identify those cellular functions that are critical for maintaining these "persister" populations as well as inhibitors that act on them. Hypomorphs have a particularly important role to play in assessing the contribution of specific targets during different stages of disease progression, including the chronic stage, in which the rate of bacillary replication is markedly reduced (Gill et al., 2009). However, the relatively frequent generation of hypomorphs of $M t b$ in which growth is able to proceed unimpeded, even under conditions of maximal transcriptional silencing (Carroll et al., 2005, 2011; Woong Park et al., 2011; Kumar et al., 2012), can complicate the genetic validation of putative persistence targets in vivo. This problem is illustrated in the case a bioA hypomorph, which, despite the requirement of BioA for growth of Mtb in vitro (Sassetti et al., 2003; Woong Park et al., 2011) and in mouse spleen (Sassetti and Rubin, 2003), showed no growth phenotype when transcriptionally silenced 

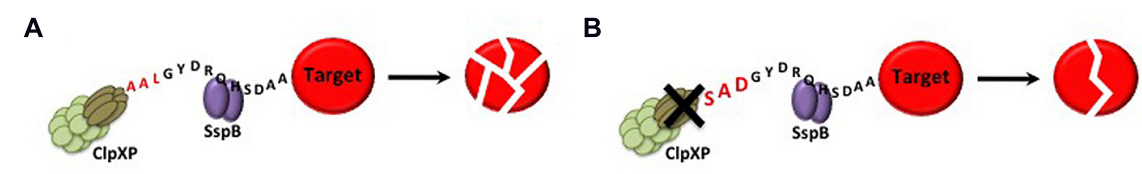

C

FIGURE 4 | Tetracycline-mediated regulated protein degradation in Msm. (A) Degradation of SsrA-tagged proteins by ClpXP is enhanced by the adapter protein, SspB. (B) Mutation of the wildtype LAA ClpX recognition site of SsrA to generate a modified DAS tag weakens the interaction of CIpXP with the aberrant protein, resulting in SspB-dependent proteolysis of the tagged protein. (C) By replacement of the native $s s p B$ promoter with a Tet-inducible promoter, SspB-mediated degradation of proteins that have been engineered to carry a modified DAS tag can be achieved upon induction with ATc. in vivo owing to residual bio $A$ expression at a level sufficient to sustain bacillary growth. In this example, mutation of the ribosome binding site upstream of bioA to create a weaker translation initiation signal resulted in dose-dependent growth of the bioA hypomorph in vitro and attenuation of growth in mouse lung (Woong Park et al., 2011), thus providing a strategy for overcoming the challenges associated with transcriptional leakiness.

An alternative strategy for addressing transcriptional leakiness is to use a modified version of the Tet-regulated system that allows simultaneous transcriptional repression of a target gene and degradation of the encoded protein, resulting in a more tightly regulated system (Kim et al., 2013; Kolly et al., 2014b). In the dual-control (DUC) system, expression of $s p B$ is regulated by TetR, and is thus repressed in the absence of ATc, while expression of the target gene is regulated by revTetR, allowing it to be expressed in the absence of ATc (Figure 5A). Upon addition of the ATc inducer, transcription of $s s p B$ proceeds, leading to Clpmediated proteolysis of the DAS+4-tagged target protein and simultaneous transcriptional silencing of the target gene through binding of revTetR to the Tet-regulated promoter (Figure 5B). The biological utility of the DUC system was confirmed by generating a BioA-DUC mutant, which showed faster kinetics of killing than achievable by transcriptional silencing or targeted proteolysis alone and also resulted in the reduced emergence of suppressors that were no longer inducer-responsive (Kim et al., 2013). A similar approach, using a modified version of the Pip/Tet-OFF regulated gene expression system (Table 1) in combination with SspB-mediated Clp proteolysis, was used to confirm the essentiality of the transketolase, Tkt, for growth of $M t b$ both in vitro and in macrophages (Kolly et al., 2014b). This system also demonstrated more rapid killing than transcriptional silencing alone (Kolly et al., 2014b), confirming the need to consider protein half-life when utilizing transcriptional silencing as a measure of target vulnerability.

Importantly, as control of the DUC system is Tet-mediated, it can also be applied in vivo to provide a powerful means of evaluating the requirement of a protein for persistence of
$M t b$ during chronic infection, with reduced risk of suppressors masking the phenotype. Kim et al. (2013) used this approach to demonstrate the absolute requirement of NAD synthetase (NadE) for non-replicating persistence of $M t b$ in vitro, and to confirm that its inactivation is bactericidal. Furthermore, the rapid decline in bacillary load in the lungs and spleens of both acute and chronically infected mice upon inactivation of NadE indicated that inhibition of NadE could kill $M t b$ in several, if not all, of the metabolic states adopted during murine infection (Kim et al., 2013), further validating this enzyme as a drug target (Boshoff et al., 2008). The DUC genetic switch has also been used to validate fructose-1,6-bisphosphate aldolase (FBA), a central enzyme in glycolysis and gluconeogenesis in $M t b$, as a persistence target (Puckett et al., 2014).

\section{Target-Based Whole-Cell Screening}

An important application of hypomorphs is in target-based whole-cell screening (TB-WCS). The rationale underlying this approach is that a reduction in the concentration of a target may render the cell hypersensitive to inhibitors that act on that target. Firstly, hypomorphs can be applied in drug susceptibility testing of individual compounds to provide biological confirmation of target specificity. The utility of TB-WCS for confirming target specificity in $M t b$ was first demonstrated by the observation that dose-dependent silencing of $d p r E 1$ resulted in hypersensitivity to benzothiazinones (Carroll et al., 2011), which are known inhibitors of DprE1 (Makarov et al., 2009). Subsequently, transcriptional silencing in $M t b$ has been used to confirm the target-specificity of PanC inhibitors designed through fragmentbased approaches (Hung et al., 2009; Abrahams et al., 2012a); PanC (Kumar et al., 2013) and DHFR (Kumar et al., 2012) inhibitors identified from high-throughput biochemical screens; a LepB inhibitor previously shown to have increased efficacy against an Escherichia coli lepB knockdown (Barbosa et al., 2002; Ollinger et al., 2012a); and hydroxyurea, as an inhibitor of the class Ib ribonucleotide reductase (Singh et al., 2015). 
A
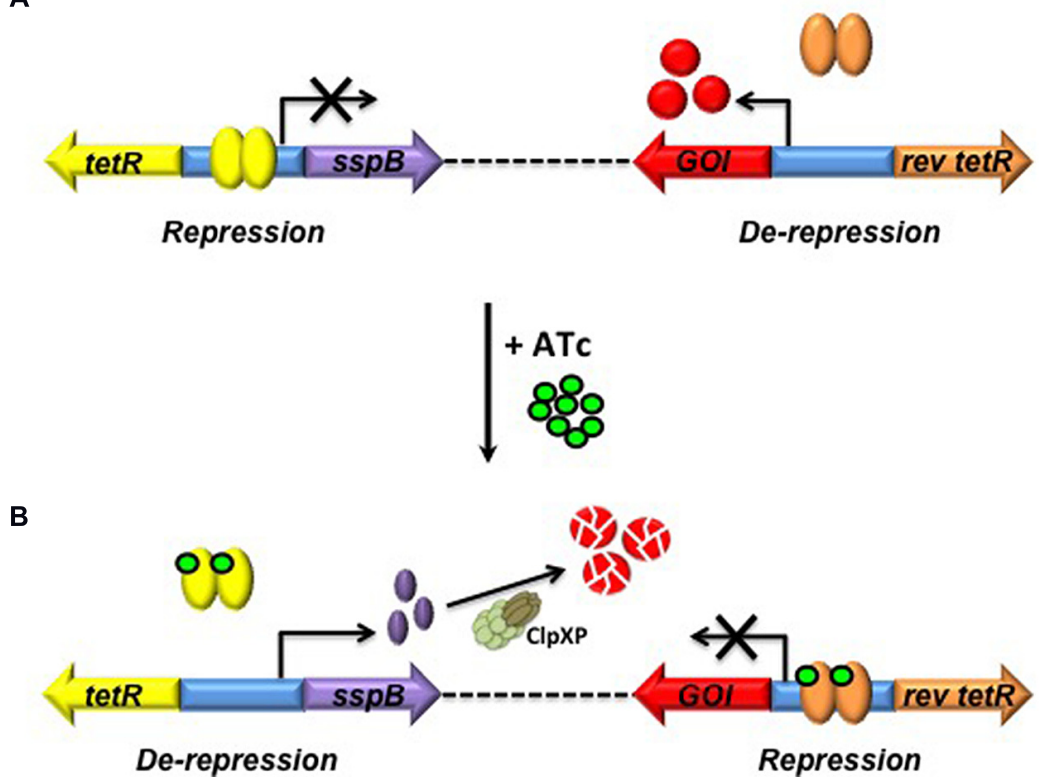

FIGURE 5 | Dual-control (DUC) genetic switch for simultaneous transcriptional silencing and degradation of the encoded protein in Mtb. (A) In the absence of ATc $s s p B$ expression is repressed by TetR, while expression of the target gene under control of revTetR proceeds. (B) Upon introduction of ATc, $s s p B$ transcription proceeds, enabling CIpXP-mediated proteolysis of the target protein and simultaneous transcriptional repression of the target gene due to binding of revTet to the Tet-regulated promoter. tetR, Tet repressor; GOI, gene of interest; rev tetR, reverse Tet repressor; ATc, anhydrotetracycline; ClpXP, protease complex mediating controlled proteolysis of the target protein (Adapted from Kim et al., 2013).
As outlined above, the discovery that the natural product, amiclenomycin, inhibits $M t b$ through covalent inactivation of BioA (Sandmark et al., 2002) has heightened interest in this particular target. However, amiclenomycin has significant liabilities, being chemically unstable, highly polar (Shi et al., 2011), and inactive against $M t b$ in in a mouse model of infection (Kitahara et al., 1975). To identify other scaffolds targeting BioA whose potency could potentially be enhanced by structure-based drug design to generate analogs with in vivo efficacy, Aldrich and colleagues (Park et al., 2015) used a BioA hypomorph to analyze the 255 hits identified by screening a 350,000 compound library for inhibitors of this enzyme. Their results were encouraging: while the majority of hits identified from the biochemical screen showed no whole-cell activity against $M t b$, several showed enhanced activity against the BioA hypomorph, confirming that these compounds were on-target in Mtb (Park et al., 2015).

Target-based whole-cell screening has also found application in enabling the direct identification of novel, cell-permeable inhibitory scaffolds with whole-cell activity against defined targets or pathways through screening of large compound libraries. The hits may include inhibitors of non-catalytic sites in an enzyme target. They may also include chemotypes that would escape detection in screens against wildtype cells by virtue of their weak inhibitory activity, thus increasing the availability of chemical matter for lead identification (Figure 3B). The feasibility of TB-WCS as a tool for identifying novel antibacterial agents was first demonstrated by the discovery of the type II fatty acid synthesis (FASII) inhibitor, platensimycin, which showed enhanced activity against Staphylococcus aureus isolates in which $f a b F$ transcript was conditionally depleted by antisensemediated interference (Wang et al., 2006; Young et al., 2006). Although TB-WCS has been used with some success in other bacteria (DeVito et al., 2002; Wang et al., 2006; Young et al., 2006; Phillips et al., 2011), it has only recently been applied in $M t b$ (Abrahams et al., 2012a). In the only example published to date, a panC hypomorph was screened against a $\sim 14,000$ compound library which led to the identification of a series of flavones that displayed increased potency against PanC-depleted cells relative to wildtype $M t b$. It was subsequently shown that these compounds do not directly inhibit PanC, despite the fact that pantothenate - the product of the PanC enzyme rescued $M t b$ from the toxic effects of the compounds (Abrahams et al., 2012a). This result confirmed that the effect of target depletion can extend beyond hypersensitization of the target itself, and may, for example, hypersensitize downstream enzymes in the same metabolic pathway to chemical inhibition through substrate depletion or diversion of metabolic flux (Palmer and Kishony, 2014). In the case of PanC, the potential for off-target hypersensitization owing to metabolic remodeling is even greater, as depletion of this target leads to CoA depletion, which may, in turn, result in hypersensitization of any number of CoAdependent enzymes. Target de-convolution in cases such as this may be challenging.

In addition to this potential complication, there are a number of other issues that should be taken into account when considering the use of TB-WCS for hit identification in TB drug discovery. Firstly, it is critical to monitor and confirm stability 
of the hypomorph during the course of the phenotypic screen as the emergence of suppressors that are no longer inducerdependent will compromise the screen. Secondly, as highlighted recently (Palmer and Kishony, 2014), an alteration in the level of expression of a target does not always manifest in a predictably altered susceptibility to a drug that acts on that target, particularly for cases in which the mechanism of action is complex. The fluoroquinolones provide an interesting case in point: these drugs act by trapping gyrase-DNA complexes, resulting in inhibition of DNA synthesis and growth arrest, followed by cell death upon release of double-stranded DNA breaks from trapped gyrase complexes (Drlica and Zhao, 1997). As such, conditional depletion of GyrA is not expected to confer fluoroquinolone hypersensitivity on mycobacteria (Wei et al., 2011). Finally, as for any phenotypic screen, the differential requirement of genes for growth under specific conditions (Pethe et al., 2010; Mak et al., 2012; Beste et al., 2013) must also be taken into account for TB-WCS. As an example, transcriptional silencing of icl1 resulted in Tet-dependent growth impairment of $M t b$ on acetate as the sole carbon source and hypersensitivity to 3-NP, but had no effect on growth of 3-NP susceptibility when cultured on glucose.

\section{Limitations in using Hypomorphs to Simulate Chemical Inhibition}

While the utility of hypomorphs in TB drug discovery has been convincingly demonstrated, variability between individual targets can sometimes make data interpretation difficult. Furthermore, the concept of genetic validation is predicated on the assumption that a perturbation in the cellular concentration of a target by transcriptional silencing or proteolytic degradation is a reasonable surrogate for the inhibitory effect of a chemical inhibitor of the target's function. In reality, the genetic vs. chemical vulnerability of a target may differ considerably as a result of a variety of factors (Wei et al., 2011). These include the local concentration of drug required for effective inhibition of the target and the time needed for the successful perturbation of the target (Lu and Tonge, 2010), both of which are likely to relate to the abundance of the target. The former is of particular significance in the case of $M t b$, whose sequestration in remote lesion compartments in the human host likely accounts for the lack of correlation observed between plasma pharmacokinetics and drug efficacy (Kjellsson et al., 2012; Lakshminarayana et al., 2015). The latter is important with regard to using genetic approaches to simulate chemical inhibition since the kinetics of chemical inhibition are significantly faster than those of gene silencing. While targeted protein degradation (Kim et al., 2011, 2013; Wei et al., 2011; Kolly et al., 2014b) has the potential to more accurately estimate target vulnerability by better simulating the kinetics of chemical inhibition (Kim et al., 2011, 2013; Kolly et al., 2014b), this approach has its own limitations: in particular, it involves modification of the C-terminus of the target protein, which could affect the structure, function and abundance of the target. The recent development of a clustered regularly interspaced short palindromic repeat interference (CRISPRi) approach to regulating gene expression in $M t b$ has the potential to overcome these limitations in that the transcriptional level of the target is maintained no modification of the expressed protein is required (Choudhary et al., 2015).

The potential disconnect between depletion of a target by genetic means and its pharmacologic inhibition is perhaps best illustrated in the case of DHFR, the target of the folate biosynthesis inhibitor, trimethoprim. Although overexpression of DHFR in both E. coli (Palmer and Kishony, 2014) and Msm (Bhat et al., 2013) was shown to result in decreased susceptibility to trimethoprim, targeted depletion of DHFR by proteolytic degradation in Msm did not phenocopy treatment with trimethoprim (Wei et al., 2011). One possible explanation is that chemical inhibition of DHFR by trimethoprim is more efficient than targeted proteolysis. Alternatively, trimethoprim could have multiple targets, requiring simultaneous inhibition of all in order to mediate growth inhibition. To distinguish these possibilities, the metabolomic consequences of DHFR depletion and treatment with sub-inhibitory concentrations of trimethoprim were compared. The production of the same metabolite profiles in both cases confirmed that DHFR is in fact the primary target of trimethoprim (Wei et al., 2011) suggesting that, in this case, even regulated degradation of the DHFR is not able to accurately recapitulate the phenotypic consequences of chemical inhibition of the enzyme.

\section{Concluding Remarks}

Given that less than a decade ago there were no robust genetic switches available for regulation of gene expression in $M t b$, remarkable progress has been made in this area. Hypomorphs provide an effective means of genetically validating novel drug targets, of comparing selected mutants in terms of vulnerability and of evaluating the downstream consequences of loss of target function. However, other factors such as cellular localization and druggability, which are critically important in determining target access by small molecule inhibitors, must also be taken into account when ranking and prioritizing novel drug targets, particularly in a pathway context. The implementation of regulated protein degradation systems and DUC genetic switches provide enhanced stringency while simultaneously enabling more rapid kinetics of inactivation, thus providing a powerful means of validating new drug targets. The increasing accessibility of 'omics approaches offer exciting prospects for combining metabolomic and proteomic analyses with genetic approaches for target validation, which, when coupled with live-cell imaging of bacilli undergoing target depletion or drug treatment, have the potential to provide key insights into the global effects that disruption of an essential cellular function can have upon bacterial metabolism and physiology. Whether the systems reviewed here have a role in validating targets in animal models other than mice, which more accurately recapitulate the pathology of human disease, remains to be seen. Nonetheless, the encouraging results obtained to date, suggest that conditional mutants are set to play an increasingly important role in early stage TB drug discovery. 


\section{Acknowledgments}

We acknowledge the TB Drug Accelerator program of the Bill and Melinda Gates Foundation, the European Community's Seventh Framework Programme (grant 260872), the South African

\section{References}

Abrahams, G. L., Kumar, A., Savvi, S., Hung, A. W., Wen, S., Abell, C., et al. (2012a). Pathway-selective sensitization of Mycobacterium tuberculosis for target-based whole-cell screening. Chem. Biol. 19, 844-854. doi: 10.1016/j.chembiol.2012.05.020

Abrahams, K. A., Cox, J. A., Spivey, V. L., Loman, N. J., Pallen, M. J., Constantinidou, C., et al. (2012b). Identification of novel imidazo[1,2a]pyridine inhibitors targeting M. tuberculosis QcrB. PLoS ONE 7:e52951. doi: 10.1371/journal.pone.0052951

Ahidjo, B. A., Kuhnert, D., Mckenzie, J. L., Machowski, E. E., Gordhan, B. G., Arcus, V., et al. (2011). VapC toxins from Mycobacterium tuberculosis are ribonucleases that differentially inhibit growth and are neutralized by cognate VapB antitoxins. PLoS ONE 6:e21738. doi: 10.1371/journal.pone. 0021738

Ahmed, W., Menon, S., Godbole, A. A., Karthik, P. V., and Nagaraja, V. (2014). Conditional silencing of topoisomerase I gene of Mycobacterium tuberculosis validates its essentiality for cell survival. FEMS Microbiol. Lett. 353, 116-123. doi: 10.1111/1574-6968.12412

Andries, K., Verhasselt, P., Guillemont, J., Gohlmann, H. W., Neefs, J. M., Winkler, H., et al. (2005). A diarylquinoline drug active on the ATP synthase of Mycobacterium tuberculosis. Science 307, 223-227. doi: 10.1126/science.1106753

Barbosa, M. D., Lin, S., Markwalder, J. A., Mills, J. A., Devito, J. A., Teleha, C. A., et al. (2002). Regulated expression of the Escherichia coli lepB gene as a tool for cellular testing of antimicrobial compounds that inhibit signal peptidase I in vitro. Antimicrob. Agents Chemother. 46, 3549-3554. doi: 10.1128/aac.46.11.3549-3554.2002

Barry, C. E., Boshoff, H. I., Dartois, V., Dick, T., Ehrt, S., Flynn, J., et al. (2009). The spectrum of latent tuberculosis: rethinking the biology and intervention strategies. Nat. Rev. Microbiol. 7, 845-855. doi: 10.1038/nrmicro2236

Begley, T. P., Kinsland, C., and Strauss, E. (2001). The biosynthesis of coenzyme A in bacteria. Vitam. Horm. 61, 157-171. doi: 10.1016/S0083-6729(01) 61005-7

Berens, C., and Hillen, W. (2003). Gene regulation by tetracyclines. Eur. J. Biochem. 270, 3109-3121. doi: 10.1046/j.1432-1033.2003.03694.x

Bertram, R., and Hillen, W. (2008). The application of Tet repressor in prokaryotic gene regulation and expression. Microb. Biotechnol. 1, 2-16. doi: 10.1111/j.1751-7915.2007.00001.x

Beste, D. J., Espasa, M., Bonde, B., Kierzek, A. M., Stewart, G. R., and Mcfadden, J. (2009). The genetic requirements for fast and slow growth in mycobacteria. PLoS ONE 4:e5349. doi: 10.1371/journal.pone.0005349

Beste, D. J., Noh, K., Niedenfuhr, S., Mendum, T. A., Hawkins, N. D., Ward, J. L., et al. (2013). 13C-flux spectral analysis of host-pathogen metabolism reveals a mixed diet for intracellular Mycobacterium tuberculosis. Chem. Biol. 20, 1012-1021. doi: 10.1016/j.chembiol.2013.06.012

Bhat, J., Narayan, A., Venkatraman, J., and Chatterji, M. (2013). LC-MS based assay to measure intracellular compound levels in Mycobacterium smegmatis: linking compound levels to cellular potency. J. Microbiol. Methods 94, 152-158. doi: 10.1016/j.mimet.2013.05.010

Bjorkelid, C., Bergfors, T., Raichurkar, A. K., Mukherjee, K., Malolanarasimhan, K., Bandodkar, B., et al. (2013). Structural and biochemical characterization of compounds inhibiting Mycobacterium tuberculosis pantothenate kinase. J. Biol. Chem. 288, 18260-18270. doi: 10.1074/jbc.M113.476473

Blanco, D., Perez-Herran, E., Cacho, M., Ballell, L., Castro, J., Gonzalez Del Rio, R., et al. (2015). Mycobacterium tuberculosis gyrase inhibitors (MGI) as a new class of antitubercular drugs. Antimicrob. Agents Chemother. 59, 1868-1875. doi: 10.1128/AAC.03913-14

Bloch, H., and Segal, W. (1956). Biochemical differentiation of Mycobacterium tuberculosis grown in vivo and in vitro. J. Bacteriol. 72, 132-141.
Medical Research Council, the National Research Foundation of South Africa and an International Research Scholar's grant from the Howard Hughes Medical Institute for financial support, and thank Digby Warner for critically reviewing the manuscript.

Blokpoel, M. C., Murphy, H. N., O’toole, R., Wiles, S., Runn, E. S., Stewart, G. R., et al. (2005). Tetracycline-inducible gene regulation in mycobacteria. Nucleic Acids Res. 33, e22. doi: 10.1093/nar/gni023

Blumenthal, A., Trujillo, C., Ehrt, S., and Schnappinger, D. (2010). Simultaneous analysis of multiple Mycobacterium tuberculosis knockdown mutants in vitro and in vivo. PLoS ONE 5:e15667. doi: 10.1371/journal.pone.0015667

Boldrin, F., Casonato, S., Dainese, E., Sala, C., Dhar, N., Palu, G., et al. (2010). Development of a repressible mycobacterial promoter system based on two transcriptional repressors. Nucleic Acids Res. 38, e134. doi: 10.1093/nar/gkq235

Boldrin, F., Ventura, M., Degiacomi, G., Ravishankar, S., Sala, C., Svetlikova, Z., et al. (2014). The phosphatidyl-myo-inositol mannosyltransferase PimA is essential for Mycobacterium tuberculosis growth in vitro and in vivo. J. Bacteriol. 196, 3441-3451. doi: 10.1128/JB.01346-13

Boshoff, H. I., Xu, X., Tahlan, K., Dowd, C. S., Pethe, K., Camacho, L. R., et al. (2008). Biosynthesis and recycling of nicotinamide cofactors in Mycobacterium tuberculosis. J. Biol. Chem. 283, 19329-19341. doi: 10.1074/jbc.M800694200

Brecik, M., Centarova, I., Mukherjee, R., Kolly, G. S., Huszar, S., Bobovska, A., et al. (2015). DprE1 is a vulnerable tuberculosis drug target due to its cell wall localization. ACS Chem. Biol. 10, 1631-1636. doi: 10.1021/acschembio.5b00237

Brown, A. C., and Parish, T. (2006). Instability of the acetamide-inducible expression vector pJAM2 in Mycobacterium tuberculosis. Plasmid 55, 81-86. doi: 10.1016/j.plasmid.2005.06.005

Carroll, P., Faray-Kele, M. C., and Parish, T. (2011). Identifying vulnerable pathways in Mycobacterium tuberculosis by using a knockdown approach. Appl. Environ. Microbiol. 77, 5040-5043. doi: 10.1128/AEM.02880-10

Carroll, P., Muttucumaru, D. G., and Parish, T. (2005). Use of a tetracyclineinducible system for conditional expression in Mycobacterium tuberculosis and Mycobacterium smegmatis. Appl. Environ. Microbiol. 71, 3077-3084. doi: 10.1128/AEM.71.6.3077-3084.2005

Choudhary, E., Thakur, P., Pareek, M., and Agarwal, N. (2015). Gene silencing by CRISPR interference in mycobacteria. Nat. Commun. 6, 6267. doi: $10.1038 /$ ncomms7267

Christophe, T., Jackson, M., Jeon, H. K., Fenistein, D., Contreras-Dominguez, M., Kim, J., et al. (2009). High content screening identifies decaprenylphosphoribose 2' epimerase as a target for intracellular antimycobacterial inhibitors. PLoS Pathog. 5:e1000645. doi: 10.1371/journal.ppat.1000645

Chuang, Y. M., Bandyopadhyay, N., Rifat, D., Rubin, H., Bader, J. S., and Karakousis, P. C. (2015). Deficiency of the novel exopolyphosphatase Rv1026/PPX2 leads to metabolic downshift and altered cell wall permeability in Mycobacterium tuberculosis. MBio 6:e2428. doi: 10.1128/mBio.02428-14

Ciulli, A., Scott, D. E., Ando, M., Reyes, F., Saldanha, S. A., Tuck, K. L., et al. (2008). Inhibition of Mycobacterium tuberculosis pantothenate synthetase by analogues of the reaction intermediate. Chembiochem 9, 2606-2611. doi: $10.1002 /$ cbic. 200800437

Cohen, J. (2013). Infectious disease. Science 339, 130. doi: 10.1126/science.339. 6116.130

Cole, S. T., and Riccardi, G. (2011). New tuberculosis drugs on the horizon. Curr. Opin. Microbiol. 14, 570-576. doi: 10.1016/j.mib.2011.07.022

Das, S., Kumar, P., Bhor, V., Surolia, A., and Vijayan, M. (2006). Invariance and variability in bacterial PanK: a study based on the crystal structure of Mycobacterium tuberculosis PanK. Acta Crystallogr. D Biol. Crystallogr. 62, 628-638. doi: 10.1107/S0907444906012728

Daugelat, S., Kowall, J., Mattow, J., Bumann, D., Winter, R., Hurwitz, R., et al. (2003). The RD1 proteins of Mycobacterium tuberculosis: expression in Mycobacterium smegmatis and biochemical characterization. Microbes Infect. 5, 1082-1095. doi: 10.1016/s1286-4579(03)00205-3

Dawson, R., Diacon, A. H., Everitt, D., Van Niekerk, C., Donald, P. R., Burger, D. A., et al. (2015). Efficiency and safety of the combination of moxifloxacin, pretomanid (PA-824), and pyrazinamide during the first 8 weeks of antituberculosis treatment: a phase $2 \mathrm{~b}$, open-label, partly randomised trial in 
patients with drug-susceptible or drug-resistant pulmonary tuberculosis. Lancet 385, 1738-1747. doi: 10.1016/s0140-6736(14)62002-x

DeVito, J. A., Mills, J. A., Liu, V. G., Agarwal, A., Sizemore, C. F., Yao, Z., et al. (2002). An array of target-specific screening strains for antibacterial discovery. Nat. Biotechnol. 20, 478-483. doi: 10.1038/nbt0502-478

Drlica, K., and Zhao, X. (1997). DNA gyrase, topoisomerase IV, and the 4-quinolones. Microbiol. Mol. Biol. Rev. 61, 377-392.

Dziadek, J. (2003). Conditional expression of Mycobacterium smegmatis $\mathrm{ftsZ}$, an essential cell division gene. Microbiology 149, 1593-1603. doi: 10.1099/mic.0.26023-0

Ehrt, S., Guo, X. V., Hickey, C. M., Ryou, M., Monteleone, M., Riley, L. W., et al. (2005). Controlling gene expression in mycobacteria with anhydrotetracycline and Tet repressor. Nucleic Acids Res. 33, e21. doi: 10.1093/nar/gni013

Ehrt, S., and Schnappinger, D. (2006). Controlling gene expression in mycobacteria. Future Microbiol. 1, 177-184. doi: 10.2217/17460913.1.2.177

Forti, F., Crosta, A., and Ghisotti, D. (2009). Pristinamycin-inducible gene regulation in mycobacteria. J. Biotechnol. 140, 270-277. doi: 10.1016/j.jbiotec.2009.02.001

Gandotra, S., Schnappinger, D., Monteleone, M., Hillen, W., and Ehrt, S. (2007). In vivo gene silencing identifies the Mycobacterium tuberculosis proteasome as essential for the bacteria to persist in mice. Nat. Med. 13, 1515-1520. doi: $10.1038 / \mathrm{nm} 1683$

Gee, C. L., Papavinasasundaram, K. G., Blair, S. R., Baer, C. E., Falick, A. M., King, D. S., et al. (2012). A phosphorylated pseudokinase complex controls cell wall synthesis in mycobacteria. Sci. Signal. 5:ra7. doi: 10.1126/scisignal.2002525

Gill, W. P., Harik, N. S., Whiddon, M. R., Liao, R. P., Mittler, J. E., and Sherman, D. R. (2009). A replication clock for Mycobacterium tuberculosis. Nat. Med. 15, 211-214. doi: 10.1038/nm.1915

Gler, M. T., Skripconoka, V., Sanchez-Garavito, E., Xiao, H., Cabrera-Rivero, J. L., Vargas-Vasquez, D. E., et al. (2012). Delamanid for multidrugresistant pulmonary tuberculosis. N. Engl. J. Med. 366, 2151-2160. doi: 10.1056/NEJMoa1112433

Gomez, J. E., and Bishai, W. R. (2000). whmD is an essential mycobacterial gene required for proper septation and cell division. Proc. Natl. Acad. Sci. U.S.A. 97, 8554-8559. doi: 10.1073/pnas.140225297

Greendyke, R., Rajagopalan, M., Parish, T., and Madiraju, M. V. (2002). Conditional expression of Mycobacterium smegmatis dnaA, an essential DNA replication gene. Microbiology 148, 3887-3900.

Griffin, J. E., Gawronski, J. D., Dejesus, M. A., Ioerger, T. R., Akerley, B. J., and Sassetti, C. M. (2011). High-resolution phenotypic profiling defines genes essential for mycobacterial growth and cholesterol catabolism. PLoS Pathog. 7:e1002251. doi: 10.1371/journal.ppat.1002251

Griffin, J. E., Pandey, A. K., Gilmore, S. A., Mizrahi, V., Mckinney, J. D., Bertozzi, C. R., et al. (2012). Cholesterol catabolism by Mycobacterium tuberculosis requires transcriptional and metabolic adaptations. Chem. Biol. 19, 218-227. doi: 10.1016/j.chembiol.2011.12.016

Griffith, K. L., and Grossman, A. D. (2008). Inducible protein degradation in Bacillus subtilis using heterologous peptide tags and adaptor proteins to target substrates to the protease ClpXP. Mol. Microbiol. 70, 1012-1025. doi: 10.1111/j.1365-2958.2008.06467.x

Guo, X. V., Monteleone, M., Klotzsche, M., Kamionka, A., Hillen, W., Braunstein, M., et al. (2007). Silencing Mycobacterium smegmatis by using tetracycline repressors. J. Bacteriol. 189, 4614-4623. doi: 10.1128/JB.00216-7

Hartkoorn, R. C., Sala, C., Neres, J., Pojer, F., Magnet, S., Mukherjee, R., et al. (2012). Towards a new tuberculosis drug: pyridomycin - nature's isoniazid. EMBO Mol. Med. 4, 1032-1042. doi: 10.1002/emmm.201201689

Hung, A. W., Silvestre, H. L., Wen, S., Ciulli, A., Blundell, T. L., and Abell, C. (2009). Application of fragment growing and fragment linking to the discovery of inhibitors of Mycobacterium tuberculosis pantothenate synthetase. Angew. Chem. Int. Ed. Engl. 48, 8452-8456. doi: 10.1002/anie.200903821

Kim, J. H., O’Brien, K. M., Sharma, R., Boshoff, H. I., Rehren, G., Chakraborty, S., et al. (2013). A genetic strategy to identify targets for the development of drugs that prevent bacterial persistence. Proc. Natl. Acad. Sci. U.S.A. 110, 19095-19100. doi: 10.1073/pnas.1315860110

Kim, J. H., Wei, J. R., Wallach, J. B., Robbins, R. S., Rubin, E. J., and Schnappinger, D. (2011). Protein inactivation in mycobacteria by controlled proteolysis and its application to deplete the beta subunit of RNA polymerase. Nucleic Acids Res. 39, 2210-2220. doi: 10.1093/nar/gkq1149
Kitahara, T., Hotta, K., Yoshida, M., and Okami, Y. (1975). Biological studies of amiclenomycin. J. Antibiot. (Tokyo) 28, 215-221. doi: 10.7164/antibiotics.28.215

Kjellsson, M. C., Via, L. E., Goh, A., Weiner, D., Low, K. M., Kern, S., et al. (2012). Pharmacokinetic evaluation of the penetration of antituberculosis agents in rabbit pulmonary lesions. Antimicrob. Agents Chemother. 56, 446-457. doi: 10.1128/AAC.05208-11

Klotzsche, M., Ehrt, S., and Schnappinger, D. (2009). Improved tetracycline repressors for gene silencing in mycobacteria. Nucleic Acids Res. 37, 1778-1788. doi: 10.1093/nar/gkp015

Kohanski, M. A., Dwyer, D. J., Wierzbowski, J., Cottarel, G., and Collins, J. J. (2008). Mistranslation of membrane proteins and two-component system activation trigger antibiotic-mediated cell death. Cell 135, 679-690. doi: 10.1016/j.cell.2008.09.038

Kolly, G. S., Boldrin, F., Sala, C., Dhar, N., Hartkoorn, R. C., Ventura, M., et al. (2014a). Assessing the essentiality of the decaprenyl-phospho-darabinofuranose pathway in Mycobacterium tuberculosis using conditional mutants. Mol. Microbiol. 92, 194-211. doi: 10.1111/mmi.12546

Kolly, G. S., Sala, C., Vocat, A., and Cole, S. T. (2014b). Assessing essentiality of transketolase in Mycobacterium tuberculosis using an inducible protein degradation system. FEMS Microbiol. Lett. 358, 30-35. doi: 10.1111/15746968.12536

Krieger, I. V., Freundlich, J. S., Gawandi, V. B., Roberts, J. P., Gawandi, V. B., Sun, Q., et al. (2012). Structure-guided discovery of phenyl-diketo acids as potent inhibitors of M. tuberculosis malate synthase. Chem. Biol. 19, 1556-1567. doi: 10.1016/j.chembiol.2012.09.018

Kumar, A., Casey, A., Odingo, J., Kesicki, E. A., Abrahams, G., Vieth, M., et al. (2013). A high-throughput screen against pantothenate synthetase (PanC) identifies 3-biphenyl-4-cyanopyrrole-2-carboxylic acids as a new class of inhibitor with activity against Mycobacterium tuberculosis. PLoS ONE 8:e72786. doi: 10.1371/journal.pone.0072786

Kumar, A., Zhang, M., Zhu, L., Liao, R. P., Mutai, C., Hafsat, S., et al. (2012). High-throughput screening and sensitized bacteria identify an M. tuberculosis dihydrofolate reductase inhibitor with whole cell activity. PLoS ONE 7:e39961. doi: 10.1371/journal.pone.0039961

Lakshminarayana, S. B., Huat, T. B., Ho, P. C., Manjunatha, U. H., Dartois, V., Dick, T., et al. (2015). Comprehensive physicochemical, pharmacokinetic and activity profiling of anti-TB agents. J. Antimicrob. Chemother. 70, 857-867. doi: $10.1093 / \mathrm{jac} / \mathrm{dku} 457$

Leblanc, C., Prudhomme, T., Tabouret, G., Ray, A., Burbaud, S., Cabantous, S., et al. (2012). 4'-Phosphopantetheinyl transferase PptT, a new drug target required for Mycobacterium tuberculosis growth and persistence in vivo. PLoS Pathog. 8:e1003097. doi: 10.1371/journal.ppat.1003097

Lederer, T., Kintrup, M., Takahashi, M., Sum, P. E., Ellestad, G. A., and Hillen, W. (1996). Tetracycline analogs affecting binding to Tn10-Encoded Tet repressor trigger the same mechanism of induction. Biochemistry 35, 7439-7446. doi: 10.1021/bi952683e

Lee, H. N., Lee, N. O., Han, S. J., Ko, I. J., and Oh, J. I. (2014). Regulation of the ahpC gene encoding alkyl hydroperoxide reductase in Mycobacterium smegmatis. PLoS ONE 9:e111680. doi: 10.1371/journal.pone.0111680

Levchenko, I., Seidel, M., Sauer, R. T., and Baker, T. A. (2000). A specificityenhancing factor for the ClpXP degradation machine. Science 289, 2354-2356. doi: $10.1126 /$ science.289.5488.2354

Ling, L. L., Schneider, T., Peoples, A. J., Spoering, A. L., Engels, I., Conlon, B. P., et al. (2015). A new antibiotic kills pathogens without detectable resistance. Nature 517, 455-459. doi: 10.1038/nature14098

Lu, H., and Tonge, P. J. (2010). Drug-target residence time: critical information for lead optimization. Curr. Opin. Chem. Biol. 14, 467-474. doi: 10.1016/j.cbpa.2010.06.176

Magnet, S., Hartkoorn, R. C., Szekely, R., Pato, J., Triccas, J. A., Schneider, P., et al. (2010). Leads for antitubercular compounds from kinase inhibitor library screens. Tuberculosis (Edinb.) 90, 354-360. doi: 10.1016/j.tube.2010.09.001

Mak, P. A., Rao, S. P., Ping Tan, M., Lin, X., Chyba, J., Tay, J., et al. (2012). A high-throughput screen to identify inhibitors of ATP homeostasis in nonreplicating Mycobacterium tuberculosis. ACS Chem. Biol. 7, 1190-1197. doi: $10.1021 / \mathrm{cb} 2004884$

Makarov, V., Manina, G., Mikusova, K., Mollmann, U., Ryabova, O., SaintJoanis, B., et al. (2009). Benzothiazinones kill Mycobacterium tuberculosis 
by blocking arabinan synthesis. Science 324, 801-804. doi: 10.1126/science. 1171583

Manjunatha, U. H., Rao, S. P. S., Kondreddi, R. R., Noble, C. G., Camacho, L. R., Tan, B. H., et al. (2015). Direct inhibitors of InhA are active against Mycobacterium tuberculosis. Sci. Transl. Med. 7, 269ra263. doi: 10.1126/scitranslmed.3010597

Marrero, J., Rhee, K. Y., Schnappinger, D., Pethe, K., and Ehrt, S. (2010). Gluconeogenic carbon flow of tricarboxylic acid cycle intermediates is critical for Mycobacterium tuberculosis to establish and maintain infection. Proc. Natl. Acad. Sci. U.S.A. 107, 9819-9824. doi: 10.1073/pnas.1000715107

Matsumoto, M., Hashizume, H., Tomishige, T., Kawasaki, M., Tsubouchi, H., Sasaki, H., et al. (2006). OPC-67683, a nitro-dihydro-imidazooxazole derivative with promising action against tuberculosis in vitro and in mice. PloS Med. 3:e466. doi: 10.1371/journal.pmed.0030466

Moolman, W. J., De Villiers, M., and Strauss, E. (2014). Recent advances in targeting coenzyme A biosynthesis and utilization for antimicrobial drug development. Biochem. Soc. Trans. 42, 1080-1086. doi: 10.1042/BST20140131

Moullan, N., Mouchiroud, L., Wang, X., Ryu, D., Williams, E. G., Mottis, A., et al. (2015). Tetracyclines disturb mitochondrial function across eukaryotic models: a call for caution in biomedical research. Cell Rep. 10, 1681-1691. doi: 10.1016/j.celrep.2015.02.034

Munoz-Elias, E. J., and McKinney, J. D. (2005). Mycobacterium tuberculosis isocitrate lyases 1 and 2 are jointly required for in vivo growth and virulence. Nat. Med. 11, 638-644. doi: 10.1038/nm1252

Murima, P., Mckinney, J. D., and Pethe, K. (2014). Targeting bacterial central metabolism for drug development. Chem. Biol. 21, 1423-1432. doi: 10.1016/j.chembiol.2014.08.020

Ojha, A. K., Mukherjee, T. K., and Chatterji, D. (2000). High intracellular level of guanosine tetraphosphate in Mycobacterium smegmatis changes the morphology of the bacterium. Infect. Immun. 68, 4084-4091. doi: 10.1128/IAI.68.7.4084-4091.2000

Ollinger, J., O’Malley, T., Ahn, J., Odingo, J., and Parish, T. (2012a). Inhibition of the sole type I signal peptidase of Mycobacterium tuberculosis is bactericidal under replicating and nonreplicating conditions. J. Bacteriol. 194, 2614-2619. doi: 10.1128/JB.00224-12

Ollinger, J., O’Malley, T., Kesicki, E. A., Odingo, J., and Parish, T. (2012b). Validation of the essential ClpP protease in Mycobacterium tuberculosis as a novel drug target. J. Bacteriol. 194, 663-668. doi: 10.1128/JB.06142-11

Palmer, A. C., and Kishony, R. (2014). Opposing effects of target overexpression reveal drug mechanisms. Nat. Commun. 5, 4296. doi: 10.1038/ncomms5296

Pandey, A. K., Raman, S., Proff, R., Joshi, S., Kang, C. M., Rubin, E. J., et al. (2009). Nitrile-inducible gene expression in mycobacteria. Tuberculosis (Edinb.) 89, 12-16. doi: 10.1016/j.tube.2008.07.007

Parish, T., Mahenthiralingam, E., Draper, P., Davis, E. O., and Colston, M. J. (1997). Regulation of the inducible acetamidase gene of Mycobacterium smegmatis. Microbiology 143(Pt 7), 2267-2276. doi: 10.1099/00221287-143-7-2267

Parish, T., Turner, J., and Stoker, N. G. (2001). amiA is a negative regulator of acetamidase expression in Mycobacterium smegmatis. BMC Microbiol. 1:19. doi: 10.1186/1471-2180-1-19

Park, S. W., Casalena, D. E., Wilson, D. J., Dai, R., Nag, P. P., Liu, F., et al. (2015). Target-Based Identification of Whole-Cell Active Inhibitors of Biotin Biosynthesis in Mycobacterium tuberculosis. Chem. Biol. 22, 76-86. doi: 10.1016/j.chembiol.2014.11.012

Payne, D. J., Gwynn, M. N., Holmes, D. J., and Pompliano, D. L. (2007). Drugs for bad bugs: confronting the challenges of antibacterial discovery. Nat. Rev. Drug Discov. 6, 29-40. doi: 10.1038/nrd2201

Pethe, K., Bifani, P., Jang, J., Kang, S., Park, S., Ahn, S., et al. (2013). Discovery of Q203, a potent clinical candidate for the treatment of tuberculosis. Nat. Med. 19, 1157-1160. doi: 10.1038/nm.3262

Pethe, K., Sequeira, P. C., Agarwalla, S., Rhee, K., Kuhen, K., Phong, W. Y., et al. (2010). A chemical genetic screen in Mycobacterium tuberculosis identifies carbon-source-dependent growth inhibitors devoid of in vivo efficacy. Nat. Commun. 1, 57. doi: 10.1038/ncomms 1060

Petrella, S., Cambau, E., Chauffour, A., Andries, K., Jarlier, V., and Sougakoff, W. (2006). Genetic basis for natural and acquired resistance to the diarylquinoline R207910 in mycobacteria. Antimicrob. Agents Chemother. 50, 2853-2856. doi: 10.1128/AAC.00244-6

Phillips, J. W., Goetz, M. A., Smith, S. K., Zink, D. L., Polishook, J., Onishi, R., et al. (2011). Discovery of kibdelomycin, a potent new class of bacterial type II topoisomerase inhibitor by chemical-genetic profiling in Staphylococcus aureus. Chem. Biol. 18, 955-965. doi: 10.1016/j.chembiol.2011.06.011

Preiss, L., Langer, J. D., Yildiz, O., Eckhardt-Strelau, L., Guillemont, J. E. G., Koul, A., et al. (2015). Structure of the mycobacterial ATP synthase Fo rotor ring in complex with the anti-TB drug bedaquiline. Sci. Adv. 1:e1500106. doi: 10.1126/sciadv.1500106

Puckett, S., Trujillo, C., Eoh, H., Marrero, J., Spencer, J., Jackson, M., et al. (2014). Inactivation of fructose-1,6-bisphosphate aldolase prevents optimal cocatabolism of glycolytic and gluconeogenic carbon substrates in Mycobacterium tuberculosis. PLoS Pathog. 10:e1004144. doi: 10.1371/journal.ppat.1004144

Raju, R. M., Unnikrishnan, M., Rubin, D. H., Krishnamoorthy, V., Kandror, O., Akopian, T. N., et al. (2012). Mycobacterium tuberculosis ClpP1 and ClpP2 function together in protein degradation and are required for viability in vitro and during infection. PLoS Pathog. 8:e1002511. doi: 10.1371/journal.ppat.1002511

Reddy, B. K., Landge, S., Ravishankar, S., Patil, V., Shinde, V., Tantry, S., et al. (2014). Assessment of Mycobacterium tuberculosis pantothenate kinase vulnerability through target knockdown and mechanistically diverse inhibitors. Antimicrob. Agents Chemother. 58, 3312-3326. doi: 10.1128/AAC.00140-14

Rengarajan, J., Bloom, B. R., and Rubin, E. J. (2005). Genome-wide requirements for Mycobacterium tuberculosis adaptation and survival in macrophages. Proc. Natl. Acad. Sci. U.S.A. 102, 8327-8332. doi: 10.1073/pnas.0503272102

Rittershaus, E. S., Baek, S. H., and Sassetti, C. M. (2013). The normalcy of dormancy: common themes in microbial quiescence. Cell Host Microbe 13, 643-651. doi: 10.1016/j.chom.2013.05.012

Rodionova, I. A., Schuster, B. M., Guinn, K. M., Sorci, L., Scott, D. A., Li, X., et al. (2014). Metabolic and bactericidal effects of targeted suppression of NadD and NadE enzymes in mycobacteria. MBio 5:e747-13. doi: 10.1128/mBio. 00747-13

Sandmark, J., Mann, S., Marquet, A., and Schneider, G. (2002). Structural basis for the inhibition of the biosynthesis of biotin by the antibiotic amiclenomycin. J. Biol. Chem. 277, 43352-43358. doi: 10.1074/jbc.M207239200

Sassetti, C. M., Boyd, D. H., and Rubin, E. J. (2003). Genes required for mycobacterial growth defined by high density mutagenesis. Mol. Microbiol. 48, 77-84. doi: 10.1046/j.1365-2958.2003.03425.x

Sassetti, C. M., and Rubin, E. J. (2003). Genetic requirements for mycobacterial survival during infection. Proc. Natl. Acad. Sci. U.S.A. 100, 12989-12994. doi: $10.1073 /$ pnas. 2134250100

Schnappinger, D., and Ehrt, S. (2014). Regulated expression systems for mycobacteria and their applications. Microbiol. Spectr. 2, 03. doi: 10.1128/microbiolspec.MGM2-0018-2013

Schnappinger, D., Ehrt, S., Voskuil, M. I., Liu, Y., Mangan, J. A., Monahan, I. M., et al. (2003). Transcriptional adaptation of Mycobacterium tuberculosis within macrophages: Insights into the phagosomal environment. J. Exp. Med. 198, 693-704. doi: 10.1084/jem.20030846

Shi, C., Geders, T. W., Park, S. W., Wilson, D. J., Boshoff, H. I., Abayomi, O., et al. (2011). Mechanism-based inactivation by aromatization of the transaminase BioA involved in biotin biosynthesis in Mycobacterium tuberculosis. J. Am. Chem. Soc. 133, 18194-18201. doi: 10.1021/ja204036t

Singh, V., Brecik, M., Mukherjee, R., Evans, J. C., Svetlikova, Z., Blasko, J., et al. (2015). The complex mechanism of antimycobacterial action of 5-Fluorouracil. Chem. Biol. 22, 63-75. doi: 10.1016/j.chembiol.2014.11.006

Spry, C., Kirk, K., and Saliba, K. J. (2008). Coenzyme A biosynthesis: an antimicrobial drug target. FEMS Microbiol. Rev. 32, 56-106. doi: 10.1111/j.1574-6976.2007.00093.x

Srivastava, D. B., Leon, K., Osmundson, J., Garner, A. L., Weiss, L. A., Westblade, L. F., et al. (2013). Structure and function of CarD, an essential mycobacterial transcription factor. Proc. Natl. Acad. Sci. U.S.A. 110, 12619-12624. doi: $10.1073 /$ pnas. 1308270110

Stallings, C. L., Stephanou, N. C., Chu, L., Hochschild, A., Nickels, B. E., and Glickman, M. S. (2009). CarD is an essential regulator of rRNA transcription required for Mycobacterium tuberculosis persistence. Cell 138, 146-159. doi: 10.1016/j.cell.2009.04.041

Stanley, S. A., Grant, S. S., Kawate, T., Iwase, N., Shimizu, M., Wivagg, C., et al. (2012). Identification of novel inhibitors of M. tuberculosis growth using whole cell based high-throughput screening. ACS Chem. Biol. 7, 1377-1384. doi: $10.1021 / \mathrm{cb} 300151 \mathrm{~m}$

Stover, C. K., Warrener, P., Vandevanter, D. R., Sherman, D. R., Arain, T. M., Langhorne, M. H., et al. (2000). A small-molecule nitroimidazopyran drug 
candidate for the treatment of tuberculosis. Nature 405, 962-966. doi: $10.1038 / 35016103$

Trauner, A., Lougheed, K. E., Bennett, M. H., Hingley-Wilson, S. M., and Williams, H. D. (2012). The dormancy regulator DosR controls ribosome stability in hypoxic mycobacteria. J. Biol. Chem. 287, 24053-24063. doi: 10.1074/jbc.M112.364851

Triccas, J. A., Parish, T., Britton, W. J., and Gicquel, B. (1998). An inducible expression system permitting the efficient purification of a recombinant antigen from Mycobacterium smegmatis. FEMS Microbiol. Lett. 167, 151-156. doi: 10.1111/j.1574-6968.1998.tb13221.x

Trujillo, C., Blumenthal, A., Marrero, J., Rhee, K. Y., Schnappinger, D., and Ehrt, S. (2014). Triosephosphate isomerase is dispensable in vitro yet essential for Mycobacterium tuberculosis to establish infection. MBio 5:e00085. doi: 10.1128/mBio.00085-14

Ventura, M., Rieck, B., Boldrin, F., Degiacomi, G., Bellinzoni, M., Barilone, N., et al. (2013). GarA is an essential regulator of metabolism in Mycobacterium tuberculosis. Mol. Microbiol. 90, 356-366. doi: 10.1111/mmi.12368

Wang, F., Sambandan, D., Halder, R., Wang, J., Batt, S. M., Weinrick, B., et al. (2013). Identification of a small molecule with activity against drug-resistant and persistent tuberculosis. Proc. Natl. Acad. Sci. U.S.A. 110, E2510-7. doi: 10.1073/pnas.1309171110

Wang, J., Soisson, S. M., Young, K., Shoop, W., Kodali, S., Galgoci, A., et al. (2006). Platensimycin is a selective FabF inhibitor with potent antibiotic properties. Nature 441, 358-361. doi: 10.1038/nature04784

Wei, J. R., Krishnamoorthy, V., Murphy, K., Kim, J. H., Schnappinger, D., Alber, T., et al. (2011). Depletion of antibiotic targets has widely varying effects on growth. Proc. Natl. Acad. Sci. U.S.A. 108, 4176-4181. doi: 10.1073/pnas.1018301108

Weiss, L. A., and Stallings, C. L. (2013). Essential roles for Mycobacterium tuberculosis Rel beyond the production of (p)ppGpp. J. Bacteriol. 195, 56295638. doi: 10.1128/JB.00759-13

WHO. (2014). Global Tuberculosis Report 2014. Geneva: World Health Organisation.
Willand, N., Dirie, B., Carette, X., Bifani, P., Singhal, A., Desroses, M., et al. (2009). Synthetic EthR inhibitors boost antituberculous activity of ethionamide. Nat. Med. 15, 537-544. doi: 10.1038/nm.1950

Wolucka, B. A. (2008). Biosynthesis of D-arabinose in mycobacteria - a novel bacterial pathway with implications for antimycobacterial therapy. FEBS J. 275, 2691-2711. doi: 10.1111/j.1742-4658.2008.06395.x

Woong Park, S., Klotzsche, M., Wilson, D. J., Boshoff, H. I., Eoh, H., Manjunatha, U., et al. (2011). Evaluating the sensitivity of Mycobacterium tuberculosis to biotin deprivation using regulated gene expression. PLoS Pathog. 7:e1002264. doi: 10.1371/journal.ppat.1002264

Xu, Z., Yin, W., Martinelli, L. K., Evans, J., Chen, J., Yu, Y., et al. (2014). Reaction intermediate analogues as bisubstrate inhibitors of pantothenate synthetase. Bioorg. Med. Chem. 22, 1726-1735. doi: 10.1016/j.bmc.2014. 01.017

Young, K., Jayasuriya, H., Ondeyka, J. G., Herath, K., Zhang, C., Kodali, S., et al. (2006). Discovery of $\mathrm{FabH} / \mathrm{FabF}$ inhibitors from natural products. Antimicrob. Agents Chemother. 50, 519-526. doi: 10.1128/AAC.50.2.519-52 6.2006

Zumla, A., Nahid, P., and Cole, S. T. (2013). Advances in the development of new tuberculosis drugs and treatment regimens. Nat. Rev. Drug Discov. 12, 388-404. doi: $10.1038 / \mathrm{nrd} 4001$

Conflict of Interest Statement: The authors declare that the research was conducted in the absence of any commercial or financial relationships that could be construed as a potential conflict of interest.

Copyright (C) 2015 Evans and Mizrahi. This is an open-access article distributed under the terms of the Creative Commons Attribution License (CC BY). The use, distribution or reproduction in other forums is permitted, provided the original author(s) or licensor are credited and that the original publication in this journal is cited, in accordance with accepted academic practice. No use, distribution or reproduction is permitted which does not comply with these terms. 